\title{
The role of astronomy and space sciences in Arab societies and cultures
}

\author{
Hamid M. K. Al-Naimiy
}

College of Sciences,

Sharjah University, PO Box 27272, Sharjah, United Arab Emirates

email: alnaimiy@gmail.com

\begin{abstract}
Astronomy, Astrophysics and Space Sciences (AASS) play an effective rôle in Sciences, Technology and Community Development. Unfortunately, a small percentage of this knowledge is actually used in teaching at schools, universities and other academic institutions in Arab countries. The challenge is to provide effective professional development for AASS educators and researchers at all levels, from elementary school to university.There is an urgent need for a better communication channels among Arab astronomers and space scientists nowadays. In this respect, the best choice is to identify in the vast cultural heritage of the Arab basin, particularly in astronomy.Building modern and good observatories, planetariums and research centres in the region jointly by Arab astronomers and space scientists is essential and will be an excellent step towards developing AASS.The aim of this paper is to show the importance of the formal and informal astronomical research and education, giving examples of possible astronomical projects, and comments of the experiences that have been carried out in a few Arab Countries. We show as well the importance of the Astronomical Societies in developing Science and Technology in the fields of AASS, and the role of these societies on the community and the country development.
\end{abstract}

Keywords. Cooperation, Algeria, Egypt, Jordan, Iraq, Lybia, UAE

\section{Introduction}

Astronomy, Astrophysics and Space Sciences (AASS) are important fields of research, study, knowledge and culture. They have been the cornerstone of both eastern and western sciences. Many Arab countries are thinking of establishing AASS research and educational centre (institute) for a variety of reasons, such as:

(a) The studies and observational techniques for research in topics of space and astronomy take advantage of the use of the most cutting edge technologies. Exceptional technological developments have been encouraged by the needs of AASS research; many space ventures are based on and are aimed at astronomy. Successful applications of topics related to AASS are introduced in many important sectors of civil life.

(b) AASS research and education topics are based on advanced physics, mathematics, sciences and technology which have a great impacts on the intellectual development of an individual to deal with modern science and technology, leading to unlimited curiosity, imagination and a sense of shared exploration and discovery (Percy 1999).

(c) AASS attracts young people to science and technology and will keep the Arab countries on track with scientific innovations and technological know-how in these countries.

(d) AASS deals with the Sun-Earth relation including the Earth's atmosphere, Heliosphere and the Earth in both time and space, and with our cosmic roots: the origin of the Sun, Earth and the elements constituting the human body and life itself. 
(e) AASS reveals a universe that is vast, varied and beautiful. Unlike most sciences, astronomy can be enjoyed as a hobby.

(f) AASS is an interdisciplinary subjects; that could greatly contribute to tertiary education in the physical sciences such as chemistry, geology, and physics. Two key areas where AASS could contribute significantly within the teaching and research of the physical sciences are the interaction of radiation with matter and the nature of information in signals. Astronomy forces education in physical and mathematical sciences away from the introspection of science for its own sake and towards science as a practical and useful tool.

(g) In some Arab states there is well-documented development in most branches of science and technology, except in AASS. It is therefore very important to start teaching topics related to AASS in schools and universities. To do so, it is essential to start by building an observatory with a sophisticated solar, optical and/or radio telescope. Although the range of $1.5-2.5 \mathrm{~m}$ optical telescope is suitable for this region, it should be equipped with state of the art technology (Al-Naimiy 2001).

These observatories could be useful for many institutions such as:

- Universities (physics, mathematics, computer sciences and engineering departments, for teaching and research purposes in undergraduate and postgraduate studies).

- Ministries and governmental institutions; such as those dealing with religious endowments (Awqaf) and Islamic affairs, communications, industry, education and tourism.

Any suggested Arab Space, Astronomy Research Centre will be an important and useful organization, because it will be designed for astronomical, space, solar physics and Earth science research, teaching and for public use, based upon the fundamental philosophy of providing every visitor with a real experience. It will also be engaged in educational activities that are linked to schools, universities or life-long education to spread astronomical observation and increase the number of astronomy amateurs in Arab countries. In addition to all of the above advantages of astronomical technologies, these observatories will be open to many visiting international astronomers, solar physicists and scientists, becoming a reputable international scientific centre of excellence.

\section{Arab astronomical heritage}

It is a well-known fact that in the middle ages, the world received important gifts from the Arabs, particularly from Mesopotamia. One of these gifts is the institution which we call today the 'Astronomical Observatory' - a place where groups of astronomers worked together for more than one generation, advancing common goals. In this sense, the concept of on astronomical observatory represents an invention of the Arabs and is one of their permanent contributions to human culture (Kopal 1983). We all know that the Babylonians were the first to name the constellations of the Zodiac, and they discovered the Saros period which they measured to be eighteen years and ten days.

A large number of bright stars and astronomical terms still carry their Arabic names (Abetti 1952) of which zenith, nadir, azimuth, alidade, almucanter are only a few examples. Names of stars such as Aldebaran, Betelgeuse, Altair, Algol are also Arabic. Several observatories were established in many Islamic places, the most important being Al Maamouni Benou Moussa, Samarra'a, Samarqand and Maraghah Observatory.

Several great astronomers lived in Mesopotamia, one of whom Al-Battani (856-929), latinized Albategnius, carried out observations of remarkable accuracy. He noticed that the longitude of the Sun's apogee had increased by 16043'since the time of Ptolemy, which implied a slow variation in the Equation of Time. He determined the length of the year to be $365 \mathrm{~d} 5 \mathrm{~h} 46 \mathrm{~m} \mathrm{24s} \mathrm{(with} \mathrm{an} \mathrm{error} \mathrm{of} 2 \mathrm{~m} \mathrm{23s).} \mathrm{Al-Battani's} \mathrm{work} \mathrm{in} \mathrm{Mesopotamia}$ 
shows like a star of the first magnitude in the firmament of astronomy, illuminating a way to the future (Kopal 1983).

With all this in the background, it is not surprising that interest in astronomy is still considered a matter of heritage in Mesopotamia. In fact, a first class observatory was built (1980-1987) in this area but unfortunately it was destroyed by the two wars (Al-Naimiy 2007).

Astronomy has influenced Arab history and culture, through its practical applications, as well as its philosophical and religious implications. This is reflected in calendars, particularly the lunar calendar (the Hijra months), mythology and a variety of art forms. Dating back 4000 years, astronomy was well advanced in Mesopotamia and Egypt, where science and technology flourished in continuous interplay with religions, substantially influencing the course of history. The achievements of Arab and Muslim scientists in Astronomy are well-known.

Islamic culture in the near and Middle East grew out of fertile ground in the preceding centuries and millennia by the Babylonians and Chaldeans (Al-Naimiy 2008).

During the period between the 11th and 15th centuries, Arab astronomy attained great heights, which resulted in important achievements and greatly influenced other sciences. For example:

(i) Arab astronomers and their discoveries in the field of solar system planetary theories, which refer to the various constructions of geometric models that represent the modern concept of the celestial spheres and planets. This was a very important episode in the history of sciences, and it has been extensively studied (Saliba 1994).

(ii) Computational mathematical astronomy particularly in solar system, called at that time (sing.zij) (Kennedy 1956), and astronomical instruments, which show the most brilliant developments in Islamic instruments made during those centuries (King 1987, King 1988, King 1999).

Many famous and great astronomers appeared during that time, such as Al-Kindy, Al-Battani, Ibn-al-Haithem, Ben Qurra, Ibn al-Shater and many others, whose names and contributions are still well known (Said 1983).

\section{Astronomical societies}

In developed industrial countries, AASS have their own societies which are strongly supported at the official level. In such countries, there are research centers, institutes, Space Agencies most are concerned with Astronomy, Space Sciences and Space observations. This is due to the fact that these countries recognize the importance of AASS in our daily life and in the future of humanity.

Satellites, for example, have transformed our vast world into a small village due to communication by means of satellite television channels, cellular phones, faxes and the internet. Satellites also are our source of continuous and instant information about the weather, environment, locations of disasters and natural crops and resources, not to mention the security, military and espionage applications. As such, these applications constitute the mother of development in the Western world as portrayed by a senior official at the U.S.A. Department of Defense in October 2007.

As a result, Astronomical Societies in the developed industrial countries receive all the necessary financial, moral and scientific support whereas their counterparts in the developing countries don't receive such desirable support. In these developing countries, such societies lack funds and attentions. In some cases these societies are banned. Even worse, in one particular Arab country, an astrophysics professor told me, "We get to know each other in conferences outside our country, not inside". 
The objectives, philosophy and goals of Astronomical Societies in the industrial countries are geared towards developing the skills of their members by providing illustrative instruments, observation equipment and astronomical photography. At times, they are even commissioned by astronomers to do some observatory tasks to observe some emerging astronomical phenomena, due to these astronomers and observatories are engaged in other scientific programs.

On the other hand, the objectives of Astronomical Societies in the developing countries are dependent on the knowledge and awareness of their governments. Thus, if they are knowledgeable enough to recognize their role in the society and the role of astronomical sciences in the country, the nation and even humanity at large, then they will have to raise the slogan, "Move astronomy from being a hobby to become institutionalized". This would mean some of the following:

(a) Rising of AASS among all sectors of the society and by all available means;

(b) Trying to include compulsory astronomy courses for students of physics, maths, geography (geophysics) and Shariah College (Astronomical Application in Islamic Sharia);

(c) Setting up an institute for AASS in one of the national Universities.

(d) Building at least an Educational Observatory to be used for academic studies and by those with interest in astronomy;

(e) Including astronomy in school curricula;

$(f)$ Focusing the efforts on schools, both students and teachers, since this sector constitutes one third of the population in each country and thus they are the reserve army of astronomy pioneers and from among them will emerge the future administrative, economic, scientific and political leaders;

(g) Sponsoring students willing to specialize in AASS;

(h) Establishing an astronomical observatory for advanced scientific purposes;

(i) Forming a high national committee to take care of all AASS affairs.

(j) Last but not least, calling for the foundation of a space agency that is capable of manufacturing satellites of all sizes and preparing the needed qualified manpower.

Astronomical Societies should aim at moving astronomy from being a hobby to a rigorous institutionalized activity. They should also highlight the weaknesses of other related institutions and regularly remind them to overcome these shortcomings.

\section{Education on AASS for Arab countries}

Because of the importance and the fast development of AASS research in the world, we need to established these topics at a small or large scale in every Arab country and should be included in the science curriculum of schools and universities, in order to increase public knowledge, understanding and appreciation of the field, as well as for educating scientists, engineers, teachers, researchers, and other science and technology personnel. There are many reasons why the related topics of AASS is useful, important and should be included in school and university curricula, at the same time there are challenges to effective teaching and learning. AASS subjects or any other subject can be taught, but there is no assurance that it will be learned (Baxter 1989, Nussbaum \& Novak (1982), Sadler 1998, Percy \& Mattei 1999). High school and undergraduate students, teachers (Woodruff et al. 1999), and the general public have deeply-rooted misconceptions about AASS topics: day and night, seasons, moon phases, celestial coordinate systems, eclipses, gravity, light, celestial motion and crescent visibility. Ahlgren (1996) and Sneider (1995) suggest strategies for teaching AASS more effectively.

It is also important to relate the curriculum to local Arabic language, culture, historical background and other needs. This is true in all parts of the Arab countries, and helps to 
meet another important challenge - reaching women, minorities, and the economically disadvantaged. In many countries, the education system favours rote memorization of lecture or textbook material. While this 'traditional' approach to teaching has some merit, it does not prepare students to develop new solutions to new problems. The problem is how to maintain the sense of awe and excitement that AASS could provide. Standard astronomy topics in the school curriculum are: day and night, seasons, moon phases, eclipses, tides, comets, planets, stars, galaxies and the Universe. Students frequently ask about peculiar celestial objects and sudden events (i.e. comets, eclipses, supernova, black holes, etc.) and sometimes about the origin and fate of the universe, the origin of life, etc.

A small number of Arab countries have good Education and Research in AASS, and some of them are planning to build a medium or large size observatory or establishing good graduate programs in Astronomy and Astrophysics. For example:

\subsection{Algeria}

Astronomy and Astrophysics is mainly done at the CRAAG (Centre de Recherche en Astronomy Astrophysique et Géophysique) in Algiers.? Related projects: Helioseimology, solar activity, solar astrolabe. Some researchers are working on the atmospheric turbulence for daytime measurements. Recently a PhD program started in Constantine University (Maymoni 2006).

\subsection{Egypt}

Research is carried out at the National Research Institute of Astronomy and Geophysics in Hellwan. Kottamia observatory operates a 2-metre optical telescope with photometers, a spectrograph and a charge-coupled device camera. The observatory was built in 1963 . The Carl Zeiss Company was involved in modernizing the optical system of this telescope (Deebes \& Heileman 1999). The Institute plans to build a radio telescope at Abu Simbel South of Egypt as part of the European (VLBI) Network, or EVN, to bridge the gap between the radio telescope in Western Europe and the radio telescope at Hartebeesthoek in South Africa (Shaltout 1999). Teaching AASS is provided in a few universities. The department of astronomy at Cairo University contributes to different fields of AASS, such as astrophysics, astro-chemistry, celestial mechanics, solar physics, and cosmology and space physics.

\section{3. $\operatorname{Iraq}$}

Iraqi astronomers established and developed AASS in 1980. They built the Iraqi National Astronomical Observatory (INAO), in the Northern part of Iraq. The INAO project was built as a West-German joint venture, in a Turn-Key contract, which included also civil work and a residential complex. Work started in 1981 and the last section of the project was finished in 1986. The observatory comprised the following telescopes: 30metre Millimetre Radio Telescope with a receiver system and its auxiliaries, and $3.5 \mathrm{~m}$ and $1.25 \mathrm{~m}$ optical telescopes.

Other astronomical observing equipment is also included such as: IR and UBVIR photometers and different types of spectrographs such as Echelle, Coudé, and Nasmyth with other needed auxiliaries. The observatory cost USD 150 million (1980 price), and built on top of the Korek mountain at 2200 metres above sea level, with a very good observational site conditions (Al-Naimiy 1986). The selection of the site was made with the help from Soviet, Egyptian, and American Astronomers. Two sites were selected (Table 1), and a site testing program accomplished in both sites. The program included the monitoring of meteorological data and seeing measurements using the Polaris Trail Telescope (PTT) 
Table 1. Astronomical observing sites

\begin{tabular}{lll}
\hline & \multicolumn{1}{c}{ Sinjar } & \multicolumn{1}{c}{ Korek } \\
\hline Altitude & $1500 \mathrm{~m}$ & $2127 \mathrm{~m}$ \\
Longitude & $41^{\circ} 45^{\prime} \mathrm{E}$ & $44^{\circ} 28^{\prime} \mathrm{E}$ \\
Latitude & $36^{\circ} 28^{\prime} \mathrm{N}$ & $36^{\circ} 35^{\prime} \mathrm{N}$ \\
\hline
\end{tabular}

Table 2. Fraction of nights with a given seeing range

\begin{tabular}{lrccc}
\hline & $<1^{\prime \prime} 1$ & $1^{\prime \prime} 1-1-1^{\prime \prime} 5$ & $1^{\prime \prime} 6-2^{\prime \prime}$ & $>2^{\prime \prime}$ \\
\hline Korek & $18 \%$ & $43 \%$ & $18 \%$ & $21 \%$ \\
Sinjar & $9 \%$ & $13 \%$ & $15 \%$ & $63 \%$ \\
\hline
\end{tabular}

Table 3. Comparison of observing sites

\begin{tabular}{|c|c|c|c|c|c|c|}
\hline Location & Best seeing & $<1^{\prime \prime} 0$ & $1^{\prime \prime} 1-1 . .5$ & $1^{\prime \prime} 6-2 . .0$ & $>2 .^{\prime \prime} 0$ & Total nights \\
\hline Junipero Sierra & $0 . .5$ & 26 & 38 & 13 & 23 & 558 \\
\hline Siero Toledo & 0.7 & 24 & 32 & 22 & 22 & 509 \\
\hline Korek & $0 . .5$ & 11 & 29 & 23 & 37 & 106 \\
\hline Kitt Peak & 0.75 & 15 & 30 & 16 & 39 & 253 \\
\hline San Pedro Martir & 0.75 & 15 & 25 & 17 & 42 & 212 \\
\hline Piper Mountain & $0 . .75$ & 9 & 30 & 20 & 42 & 164 \\
\hline Mt. McKinley & $0 . .75$ & 2 & 32 & 20 & 46 & 50 \\
\hline Sinjar & 0.75 & 1 & 19 & 21 & 59 & 100 \\
\hline Flagstaff & $1^{\prime \prime} 0$ & 1 & 5 & 29 & 65 & 80 \\
\hline
\end{tabular}

method developed by Prof. M.F. Walker from the University of California at Santa Cruz. The programme was carried out by Iraqi personnel assisted by a group from the Royal Greenwich Observatory (RGO). PTT plates were processed and evaluated by Walker.

Table 2 shows seeing measurements for both sites (October 1977 - January 1978). According to these results, Mt. Korek was selected, but site testing continued for a full year (October 1977 - August 1978). Results were then compared with other international sites (Table 3 ).

Unfortunately, this observatory was damaged during the two wars (1980 and 1991). Recently we had some correspondence with the local scientists and Kurdistan Government for rebuilding the observatory at Korek Mountain. A solar telescope and 5-6 meter optical telescope of modern design have been suggested which probably fit in the 3.5-meter dome.

\subsection{Arab Union for Astronomy and Space Sciences (AUAASS)}

This union established in Amman in 1998. More than 250 astronomers and scientists from 19 Arab countries are members in the Union. The aim of the union is to develop AASS in Arab countries through conferences, meetings, publications, joint research projects, etc. with the cooperation of international AASS institutions (Al-Naimiy \& Konsul 2001). Recently the AUAASS council decided to involve heavily in the activities of the IYA 2009 .

\subsection{Libyan Arab Jamahiriya}

Libyan space centre start on 2005 building a 2-metre robotic optical telescope. Observations and research are being carried out for choosing a suitable site out of three being 
studied. A training program for astronomers and technical engineers in process and the telescope is in manufacturing process.

\section{AASS cooperation between Arab countries}

Enhancing AASS facilities and research centres in Arab countries require cooperation with international institutions in general and among many Arab universities and organizations in particular. Establishing good AASS in any region cannot be accomplished without strong cooperation between different communities. In Arab countries, everything for AASS development is available (such as budget, personnel, sites, environments, etc) the only thing missing is cooperation and scientific support from international organizations and scientists.

Building good, modern observatories in the region jointly by Arab astronomers and scientists is essential and will be an excellent step toward developing astronomy and astrophysics, particularly when the Astronomical stations and the observatories contain modern size solar and optical telescopes with their auxiliaries, besides a millimetre radio telescope to be part of any International VLBI system or any international network system. Keeping in mind that there are good sites for observatories, such as mountains in different parts of Arab countries, particularly in those places were observations can be made of both southern and northern parts of the sky. It might be useful if the observatories were to be built in collaboration between the Arab and International astronomers.

\section{Conclusions}

In the Arab region, it is important to establish two main Education and Research Centres in fields of AASS. These centres could currently be Associated with this initiative: the Arab Space City (ASC), and the Arab Astronomical Facility (AAF). The AAF would be oriented towards supporting higher education and university level astronomical and space science research and development. Its centre-piece would be the optical and radio observatories, located on a suitable astronomical site, with a major support centre comprising Assembly and lecture halls appropriate to hosting sizable conferences as well as laboratory and classroom facilities, which would be built in Association with or near to the already established infrastructure of a University.

The ASC can be established in the vicinity of the capital city in any of Arab country, and would focus on providing the general public with awareness of the importance of science and technology for their own lives and the lives of their children, and for inspiring younger students to a career in AASS. These themes would be developed by introducing the history of Arabic and ancient Middle-Eastern discoveries and contributions to the science of astronomy up to and including the present epoch, via the Astronomy and Space Museum, the Interactive Astronomical Image Gallery, the Planetarium and the continuous solar activities show experience, using a range of topics, changing according to monthly themes, to bring many viewers back. With a 500-600 seat planetarium, for example, there would be every reason to encourage classroom groups of school children from virtually every nation of the Arab World to come to ASC to have this experience. It would be such an experience to see the wonders of the universe, and participate in personal hands-on space and astronomy activities, space-camps, flight simulators, observe real-time solar activity with a dedicated solar imaging telescope, see special exhibits depicting current space activities such as comet sightings, asteroid missions, lunar and 
Mars exploration activities, space satellites, activities on the International Space Station. There would always a connection to the activities at the suggested AAF. This aspect of the ASC would both stimulate and educate the public at large, and give rise to new generations of students to populate the science curriculum of the Arab Universities and indeed all of the nation's colleges and universities as well. Such an arising awareness and affinity with AASS and their applications coupled with the Islamic background most fulfilling future for all the peoples of region, the entire Arab World, a vision of harmony combined with maximum personal achievements for everyone, a vision which we all dream of together, deep within. In a country encouraging the development of science and technology, it is natural to build a large and important AASS centres. One of the main goals of the space initiative is to promote the Arab astronomy heritage and to become a core scientific research centre not only for Arab countries, but for all the Middle East and perhaps for Asia. It will also provide life information for the space sciences to the public community. The project will concentrate on scientific research, education, learning, knowledge and scientific entertainment. This will be through the exploration of the universe and the discovery of the cosmos and celestial objects, which gives great achievements to the human community.

\section{References}

Abetti, G. 1952, The History of Astronomy (New York: Henry Schuman)

Al-Naimiy, H. M. 1986, Astrophysics and Space Science, 118, 51

Al-Naimiy, H. M. 2001, Teaching of Astronomy in Asian-Pacific Region Bulletin, 17, 13

Al-Naimiy, H. M 2007, Bull. Astro. Soc. India, 35, 727

Al-Naimiy, H. M. 2007, International Symposium on Solar Physics and Solar Eclipses, R. Ramelli, O. M. Shalabiea, I. Saleh, and J. O. Stenflo (eds) (Locarno: Istituto Ricerche Solari Locarno), p. 143

Al-Naimiy, H. M., and Konsul, K. 2001, Teaching of Astronomy in Asian-Pacific Region Bulletin, 17,27

Ahlgren, A. 1996, in Astronomy Education: Current Developments, Future Coordination, eds. J. R. Percy, ASP Conference Series, 89, 26

Baxter, J. 1989, Int. J. Sci. Ed., 11, 502

Deebes, H. A. \& Heileman, W. 1999, Cieux Africains/African Skies, 4, 7

Kennedy, E. S. 1956, Transactions of the American Philosophical Society, 46, part 2, 123

King, D. A. 1987, in From Ancient Omens to Statistical Mechanics: Essays on the Exact Sciences Presented to Asger Aaboe, eds. J. L. Berggren and B. R.Goldstein, Acta Historica Scientiarum Naturalium et Medicinalium, Bibliotheca Universitatis Hauniensis (Copenhagen: University Library), Vol. 39, 121

King, D. A. 1988, in Essays on Islamic Culture in Honour of Richard Baly Winder (New York: New York University Press), 153

King, D. A. 1999, in Innovation and Tradition in Islamic Science / Islamic Philosophy Theology Es Science, eds. H. Daiber \& D. Pingree, (London: Al-Furqan Islamic Heritage Foundation), Vol. 36

Kopal, Z. 1986, in Islamic Cultural Identity and Scientific-Technological Development, K. Gottstein (ed) (Baden-Baden: Nomos Verlag)

Maymoni, J. 2006, personal communication

Nussbaum, J. \& Novak, J. 1982, Instructional Sci., 11, 183 ???

Percy, J. R. 1999, Teaching of Astronomy in Asian-Pacific Region Bulletin, 15, 41

Percy, J. R., and Mattei, J. A. 1999, Teaching of Astronomy in Asian-Pacific Region, Bulletin, 15,51

Sadler, P. M. 1998, J. Res. Sci. Tchg., 35, 265

Shaltout, M. A.M. 1999, Cieux Africains/African Skies, 4, 10 
Said, H. M. 1983, Personalities Noble, Glimpses of Renowned Scientists and Thinkers of Muslim Era), (Karachi: National Science Council of Pakistan)

Saliba, G. 1994, A History of Arabic Astronomy (Planetary Theories during the Golden Age of Islam) (New York: New York University Press)

Sneider, C. 1995, in The Universe at your Fingertip, ed. A. Fraknoi, (San Francisco: Astronomical Society of the Pacific)

Woodruff, E., Chandra, N., Kalchman, M., \& Percy, J. R. 1999, Paper Presented at the Universe in Your Classroom, Toronto, Canada, 3 July 1999 